\title{
RANKL and RANK in extracellular vesicles: surprising new players in bone remodeling
}

\author{
L. Shannon Holliday ${ }^{1,3}$, Shivani S. Patel ${ }^{1}$, Wellington J. Rody, Jr ${ }^{2}$ \\ 'Department of Orthodontics, University of Florida College of Dentistry, Gainesville, FL 32610, USA. \\ 2Department of Orthodontics and Pediatric Dentistry, Stony Brook School of Dental Medicine, Stony Brook, NY 11794, USA. \\ ${ }^{3}$ Department of Anatomy \& Cell Biology, University of Florida College of Medicine, Gainesville, FL 32610, USA.
}

Correspondence to: Prof. L. Shannon Holliday, PhD, Department of Orthodontics, University of Florida College of Dentistry, 1600 SW Archer Road, Gainesville, FL 32610, USA. E-mail: sholliday@dental.ufl.edu

\begin{abstract}
How to cite this article: Holliday LS, Patel SS, Rody WJ, Jr. RANKL and RANK in extracellular vesicles: surprising new players in bone remodeling. Extracell Vesicles Circ Nucleic Acids 2021;2:18-28. https://dx.doi.org/10.20517/evcna.2020.02
\end{abstract}

Received: 10 Nov 2020 First Decision: 5 Jan 2021 Revised: 11 Jan 2021 Accepted: 14 Jan 2021 Available online: 30 Mar 2021

Academic Editor: Y. Peng Loh Copy Editor: Yue-Yue Zhang Production Editor: Xi-Jun Chen

\begin{abstract}
Receptor activator of nuclear factor kappa B-ligand (RANKL), its receptor RANK, and osteoprotegerin which binds RANKL and acts as a soluble decoy receptor, are essential controllers of bone remodeling. They also play important roles in establishing immune tolerance and in the development of the lymphatic system and mammary glands. In bone, RANKL stimulates osteoclast formation by binding RANK on osteoclast precursors and osteoclasts. This is required for bone resorption. Recently, RANKL and RANK have been shown to be functional components of extracellular vesicles (EVs). Data linking RANKL and RANK in EVs to biological regulatory roles are reviewed, and crucial unanswered questions are examined. RANKL and RANK are transmembrane proteins and their presence in EVs allows them to act at a distance from their cell of origin. Because RANKL-bearing osteocytes and osteoblasts are often spatially distant from RANK-containing osteoclasts in vivo, this may be crucial for the stimulation of osteoclast formation and bone resorption. RANK in EVs from osteoclasts has the capacity to stimulate a RANKL reverse signaling pathway in osteoblasts that promotes bone formation. This serves to couple bone resorption with bone formation and has inspired novel bifunctional therapeutic agents. RANKL- and RANK- containing EVs in serum may serve as biomarkers for bone and immune pathologies. In summary, EVs containing RANKL and RANK have been identified as intercellular regulators in bone biology. They add complexity to the central signaling network responsible for maintaining bone. RANKL- and RANK-containing EVs are attractive as drug targets and as biomarkers.
\end{abstract}


Keywords: Cell signaling, osteoclast, osteoblast, osteocyte, exosome, microvesicle, coupling factor, apoptotic vesicle

\section{INTRODUCTION}

Receptor activator of nuclear factor kappa B-ligand (RANKL), its receptor RANK, and the RANKL-binding decoy receptor osteoprotegerin are members of the tumor necrosis factor (TNF) and TNF receptor superfamilies ${ }^{[1]}$. In the late 1990s, these proteins were identified as essential for regulating the formation of osteoclasts and thus for bone remodeling ${ }^{[2,3]}$. Mice in which RANKL was knocked out had few or no osteoclasts, the hematopoietic cells specialized to resorb bone, and were severely osteopetrotic ${ }^{[4]}$. In addition, these mice had immune cell abnormalities and lacked certain lymph nodes. Female RANKL knockout mice died before maturity as a consequence of defective breast development ${ }^{[5]}$. Mice in which osteoprotegerin was knocked out were osteoporotic ${ }^{[2]}$. This led to the hypothesis that bone resorption was a function of the ratio of RANKL to osteoprotegerin.

These regulatory molecules were identified due to efforts to find the "osteoclast differentiation factor". Indirect evidence had suggested that there might be a specific regulatory molecule responsible for directing hematopoietic stem cells to differentiate into osteoclasts. Since bone diseases, in particular osteoporosis and metastatic bone cancer, are major clinical problems that are associated with excess bone resorption by osteoclasts, it was reasoned that if such a specific regulatory molecule existed, it would be an extremely attractive target for drug development ${ }^{[6,7]}$. Groups from Amgen and Snow Brand Milk Products concurrently identified RANKL (called "osteoprotegerin-ligand" by the Amgen group and "osteoclast differentiation factor" by the group from Snow Brand) as the osteoclast differentiation factor ${ }^{[2,3]}$. The nomenclature was later resolved to RANKL. Osteoprotegerin, which binds RANKL and blocks its interaction with RANK, and thus osteoclast formation, has not been developed directly into a therapeutic agent because it is promiscuous, binding other TNF superfamily members, and has a short half-life in circulation $^{[6]}$. Amgen developed a humanized monoclonal antibody directed against RANKL (denosumab), which has become a major therapeutic for the treatment of osteoporosis (Prolia) and bone cancer (Xgeva) ${ }^{[7-9]}$. Although RANKL, RANK, and osteoprotegerin have roles in the immune system, the use of denosumab as a therapeutic has had relatively few off target effects.

Extracellular vesicles (EVs) include exosomes and microvesicle (also known as ectosomes) ${ }^{[10,11]}$. Exosomes are released from cells as cytosolic multivesicular bodies fuse with the plasma membrane. The multivesicular bodies are formed by inward budding of the bounding membrane of cytosolic vesicles. Microvesicles are formed by direct budding from the plasma membrane. Evidence suggests that there is considerable overlap in the composition and size range of exosomes and microvesicles. For this reason, the International Society for Extracellular Vesicles recommends using the term EV to describe these vesicles unless there is a clear evidence that the EVs are exosomes or microvesicles ${ }^{[12]}$. In this article we will use the term EV, though various nomenclatures were used in the original reports. A third type of EV is apoptotic vesicles that form during apoptosis, but these are larger and are usually separated from exosomes and microvesicles during standard isolations. During the past decade, many studies have supported the hypothesis that EVs serve as intercellular regulators ${ }^{[13]}$.

The discovery of RANKL in EVs in $2015^{[14]}$, and RANK in EVs in $2016^{[15]}$, together with other recent advances in the understanding of bone biology, particularly the finding that most RANKL that stimulates bone resorption is from osteocytes ${ }^{[16-20]}$, has opened up new lines of study [Figure 1]. Incorporation of these transmembrane proteins into EVs allows each to be, as needed, either a receptor or a ligand, serving as an 


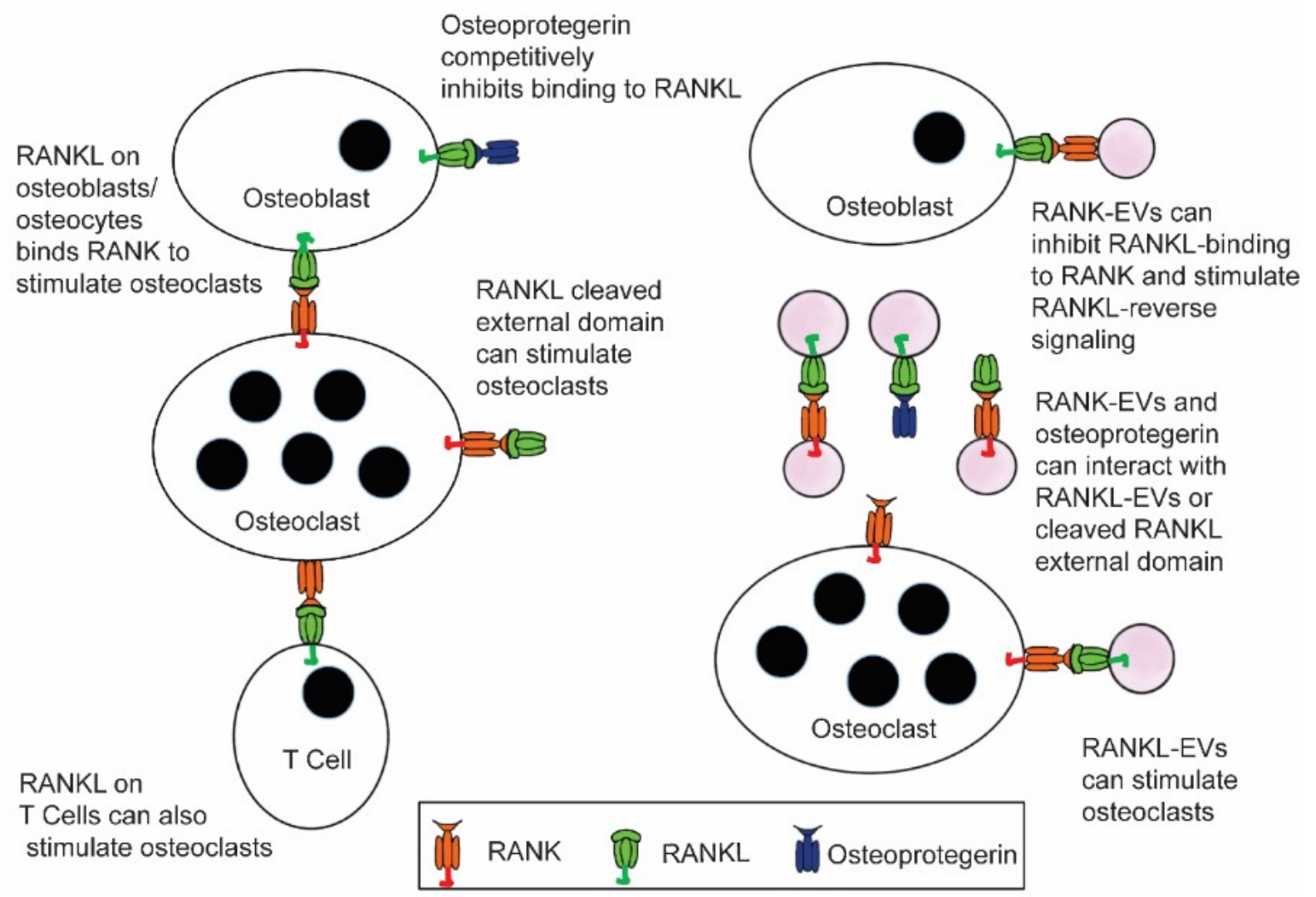

Figure 1. Extracellular vesicles containing RANKL or RANK add increased complexity to the RANKL/RANK/osteoprotegerin signaling network that is at the core of bone biology. Until recently, RANKL stimulation of RANK to stimulate osteoclast formation and bone resorption by osteoclasts, and the ability of osteoprotegerin to bind RANKL and competitively inhibit this signaling, were considered the primary features of this network. Now data suggest that RANKL EVs can stimulate osteoclast formation and bone resorption through RANK stimulation, and RANK-EVs bind to RANKL on osteoblasts to stimulate RANKL reverse signaling and bone formation. The latter serves to couple bone resorption and bone formation. It is also possible that RANK-EVs can bind RANKL or RANKL-EVS and competitively inhibit their stimulation of RANK on osteoclasts. RANKL-EVs could serve as competitive inhibitors of RANK-EV's stimulation of RANKL reverse signaling.

elegant and parsimonious evolutionary solution for regulating the coupling of bone resorption and bone formation. As this work develops, it promises deeper understanding of how bone is remodeled, and new therapeutic approaches to treat bone disease. However, numerous unanswered questions are open in this field of research.

\section{RANKL-EVS}

\section{Osteoblast EVs: long range stimulation of osteoclastic bone resorption}

RANKL is expressed by osteoblasts, osteocytes, T cells, B cells, and various other tissues during development ${ }^{[4,19,20]}$. It is also found in the brain in the lateral septal nucleus ${ }^{[21]}$, and astrocytes and microglia contain RANK and respond to RANKL ${ }^{[2,223]}$. RANKL is a transmembrane protein that can also act as a receptor ${ }^{[24]}$. RANKL exists in cells as a homotrimer. The binding site for RANK (also a homotrimer) on RANKL is formed by elements of two adjacent RANKL molecules ${ }^{[25]}$. Until the discovery of RANKL in EVs, two forms of RANKL were known in biology, the intact membrane bound form, and a soluble form cleaved from the plasma membrane by exoproteases/sheddases including TNF- $\alpha$ converting enzyme, several a disintegrin and metalloproteinases, and matrix metalloproteinases ${ }^{[26-28]}$. A recent study showed that in mice where RANKL was altered to prevent the action of exoproteases, normal bone physiology was not 
perturbed, although the mice were more susceptible to cancer invasion ${ }^{[20]}$.

Data suggesting regulatory RANKL in EVs was first described in an in vitro system ${ }^{[14]}$. EVs isolated from a stromal/osteoblastic cell line (UAMS-32P) contained RANKL and were able to stimulate differentiation of RAW 264.7 cells into osteoclast-like cells. Immunogold transmission electron microscopy indicated that the EVs containing RANKL were large (200 nm or more), suggesting that they may be microvesicles. While this report utilized cell lines, it provided the first suggestion of functional RANKL in EVs.

These data were confirmed and extended to show that EVs released by primary osteoblasts (isolated from mouse calvaria) produced RANKL-containing EVs that stimulated primary mouse monocytes to differentiate into osteoclasts ${ }^{[30]}$. These EVs were also large, 100-200 $\mathrm{nm}$ in diameter, suggesting that they may be microvesicles. In addition, these RANKL-containing EVs triggered osteoclast formation in vivo when injected into transgenic mice in which RANKL was knocked out. By using osteoblast EVs loaded with the fluorophore chloromethyl fluorescein diacetate, it was shown that the osteoblast-derived EVs could fuse with target cells and deliver the luminal contents to the cytosol. It was then demonstrated that RANKLcontaining EVs could be used to deliver anti-osteoclastic drugs to osteoclasts in vivo. Overactive osteoclasts were stimulated by treatment of mice with retinoic acid. These mice were then injected with RANKLcontaining EVs from osteoblasts in which the osteoclast inhibitors, zoledronic acid (which blocks prenylation pathways) or dasatinib (which blocks tyrosine kinases including c-src) had been incorporated. The therapeutic efficacy of the EVs was demonstrated by the reduction of the overstimulation of osteoclasts, caused by retinoic acid, measured by both a serum marker of bone resorption and counts of osteoclasts and apoptotic osteoclasts in bone sections ${ }^{[30]}$. In a larger sense, these results suggest that in addition to stimulating RANK on the surface of the osteoclasts, the RANKL-EVs fused with and delivered the contents of the lumen of the EVs (osteoclast inhibiting pharmaceuticals) to the target cells. While in this case, the lumens contained zoledronic acid or dastinib, it suggests that under physiologic conditions, regulatory agents found in the EV lumen (microRNAs for example) are targeted to the cytosol of osteoclasts. Characterization of the composition of RANKL-EVs shed by osteoblasts will be required to identify other regulatory signals that may be present.

Recently, a zebra-fish model using fluorescently-tagged proteins for direct visualization of EVs was used to demonstrate that osteoblasts release RANKL-containing EVs that stimulate osteoclast formation in vitro, and also deliver luminal contents to osteoclasts in vivo ${ }^{[31]}$. These data confirmed the results of the studies in mammalian cells and suggest that the involvement of RANKL-containing EVs in bone signaling has ancient evolutionary origins.

Studies have suggested that EVs from osteoblasts and osteocytes are involved in communication with each other, with mesenchymal stem cells, and with myoblasts ${ }^{[32]}$. Osteoblast EVs contain various microRNAs, but the receptor-ligand binding interactions that dock the EVs have not been identified. It is also not clear whether microRNAs or other type of RNAs carried in EVs are acting in these regulatory pathways.

\section{RANKL in osteocyte EVs}

Osteocytes have been shown to release regulatory $\mathrm{EVs}^{[33-38]}$ and RANKL was shown to be released by in EVs from osteocytes in response to mechanical force ${ }^{[34]}$. However, it has not yet been shown that RANKLcontaining EVs shed by osteocytes stimulate biologically relevant osteoclast formation. It has emerged that most (90\%) of the RANKL stimulation of osteoclasts in vivo in mammals is by RANKL derived from osteocytes $^{[17]}$. These cells are a final differentiation step in the osteoblast lineage and are the most abundant cells in bone, inhabiting lacunae that weave through the bone. It is not clear how RANKL on the surface of 
osteocytes would be accessible to osteoclasts and their precursors. One possibility is that soluble RANKL cleaved by proteases might serve to stimulate distant osteoclasts, but recent data indicating soluble RANKL is not required for normal bone physiology suggest that this is not the case $\mathrm{e}^{[29]}$. It is attractive to hypothesize that RANKL-containing EVs released from osteocytes have the ability to stimulate spatially distant osteoclasts.

\section{RANK-EVS}

\section{Osteoclast EVs: RANKL reverse signaling}

RANK was first identified in EVs in vitro in efforts to identify the basis of differential regulatory activities of EVs released by pre-osteoclasts compared with EVs released from mature osteoclasts ${ }^{[15]}$. Several differentially expressed proteins were detected, and mass spectrometry identified RANK to be relatively abundant in EVs from osteoclasts, but not in EVs from precursors. This result was confirmed by immunoblots and by immunogold labeling of EVs from osteoclasts visualized in negative stained transmission electron microscopy. The electron microscopy indicated that RANK in EVs was concentrated in a small subset of the total EVs shed by osteoclasts. The size of the EVs in which RANK was detected was about 50-nm in diameter, which is consistent with the RANK-containing EVs being exosomes. Data were also presented that suggested that the RANK-containing EVs inhibited osteoclast formation in calcitriolstimulated mouse marrow cultures by a paracrine mechanism. It was suggested that RANK-EVs might act as competitive inhibitors of the RANKL-RANK interaction in the manner of osteoprotegerin ${ }^{[15,39]}$.

The publication showing RANK in EVs appeared concurrently with two other article describing regulatory EVs released by osteoclasts ${ }^{[4,41]}$. Both of these reports found that EVs from osteoclasts delivered microRNA214-3p to osteoblasts and consequent reduction of target mRNA translation inhibited osteoblast formation and function. One group reported that targeting of the EVs from osteoclasts to osteoblasts was achieved by interaction between semaphorin $4 \mathrm{D}$ on the osteoclast EVs with plexinB1 on the osteoblast ${ }^{[40]}$. The semaphorin $4 \mathrm{D}$-containing EVs then fused with the osteoblast to release luminal microRNA 214-3p into the cytosol where it inhibited translation of the mRNA coding for activating transcription factor 4 . This resulted in reduced osteoblast formation and bone formation ${ }^{[40]}$. The second study found that osteoclast EVs bound to osteoblasts through ephrinA2 binding to ephrin receptor on the osteoblasts. They also reported that osteoblasts were inhibited due to the introduction of microRNA 214-3p into the osteoblast cytosol. Both of these studies found EVs from osteoclasts to be inhibitors of osteoblasts ${ }^{[40,41]}$. These studies were not consistent with EVs from osteoclasts serving as "coupling factors" that signal to balance bone formation with bone resorption.

The initial finding of RANK in EVs was subsequently confirmed by several groups ${ }^{[42-44]}$. Ikebuchiet al. ${ }^{[24]}$ greatly expanded the understanding of RANK-containing EVs in an article in Nature. They showed that when RANK-containing EVs bind RANKL on osteoblasts, a RANKL reverse signaling pathway is stimulated that triggers pre-osteoblastic cells, which do not form bone, to differentiate and form bone. This reverse signaling pathway acted through phosphatidylinositol 3-kinase, mammalian target of rapamycin and runt-related transcription factor 2. They hypothesized that the RANK-containing EVs would act as "coupling factors" that could promote bone formation to replace bone resorbed by osteoclasts ${ }^{[45,46]}$. They demonstrated in vivo that RANK-EVs promoted bone formation in the calvarial critical size bone defect model. In an exciting addition, they found that certain cross-linked anti-RANKL antibodies mimicked RANK-containing EVs to stimulate RANKL reverse signaling. These antibodies were tested in an in vivo rodent model of postmenopausal osteoporosis. They blocked the ability of RANKL to stimulate RANK, like osteoprotegerin, and thus blocked bone resorption. Unlike osteoprotegerin, they also stimulated RANKL reverse signaling and bone formation. This provided a proof-of-concept for a new type of bifunctional 
therapeutic agent that may have the potential to bring about transformative changes to the way osteoporosis and other bone diseases are treated.

\section{Regulation of RANK-EVs release by osteoclasts}

It is attractive to hypothesize that the shedding of RANK-containing EVs is regulated. One line of evidence supporting this idea is the finding that osteoclasts resorbing bone released 5-times more RANK than the same cells resorbing dentin, the mineralized tissue that makes up the bulk of a tooth ${ }^{[47]}$. The differential release of RANK in EVs was accompanied by differential release of numerous other components as determined by quantitative high-resolution liquid chromatograph-mass spectrometry. The difference in RANK-containing EV shedding observed in this study could have been driven by matrix-related factors. This is biologically plausible in that tooth resorption is not coupled to bone formation. Moreover, if coupled bone formation occurred associated with tooth resorption, it could lead to fusion of the alveolar bone to the tooth structure, a pathological process named ankylosis. It is of interest to identify the factors in bone that trigger RANK-containing EV release, or perhaps the factors that suppress release when the cells are resorbing dentin. Although the two mineralized tissue share many components, previous studies show that there are compositional differences ${ }^{[48-50]}$.

What about other factors? For example, do specific cytokines known to regulate osteoclast formation and activity also activate, or suppress RANK-EV release? Might mechanical stress, which favors bone formation in vivo ${ }^{[5,52]}$, stimulate RANK-EV shedding in the same way as it does with osteocytes? At a more fundamental level, what mechanisms are involved in packaging RANK into EVs? For example, are receptors incorporated into EVs, in this case exosomes, as a pathway that is part of the general receptor recycling process [Figure 2]? RANK recycling after internalization from the plasma membrane has been shown to be controlled by the retromer complex ${ }^{[53]}$. Reduction in the vps35 component of the retromer leads to more RANK on the osteoclast surface, and increased bone resorption that is uncoupled with bone formation in mice leading to osteoporosis ${ }^{\left[{ }^{[3]}\right]}$. We suggest that RANK recycling may simultaneously regulate the number of RANK molecules on the osteoclast cell surface that are available for stimulation by RANKL, and the number of RANK molecules loaded into exosomes. Regulation of the recycling process could serve as an osteoclast-centered rheostat regulating the amount of osteoclastic bone resorption and the relative amount of bone formation coupled to the bone resorption.

\section{RANK-EVs in apoptotic bodies from osteoclasts}

A number of recent studies have suggested a change in paradigm; apoptotic bodies/vesicles may serve not only as easily handled materials for cells tasked with cleaning the dead cells remains, but also as regulatory signal $^{[13]}$. In particular, apoptotic vesicles have been shown to produce pro-inflammatory signals. Apoptosis of osteoclasts typically precedes the recruitment and differentiation of osteoblasts in a bone remodeling unit ${ }^{[45]}$. Ma et al. ${ }^{[54]}$ explored whether apoptotic bodies might contain RANK and stimulate reverse RANKL signaling in the same way and RANK-containing EVs. They found that when osteoclasts were grown in culture and stimulated to undergo apoptosis with alendronate, apoptotic bodies recovered from the conditioned media contained RANK. These apoptotic vesicles were able to stimulate RANKL-reverse signaling and mineralization by osteoblasts as had been described by RANK-containing EVs ${ }^{[24]}$. The stimulation was blocked by the inclusion of recombinant soluble RANKL. Although this is an intriguing in vitro result, questions remain regarding whether this is a true physiologic mechanism. For example, when alendronate is given in vivo, it binds bone and reduces bone resorption by triggering osteoclasts that begin to resorb the treated bone to undergo apoptosis ${ }^{[55]}$. If apoptotic bodies were a significant anabolic signal, alendronate should be a potent bone anabolic agent. Instead, alendronate is able to reduce bone loss, but not stimulate increases in bone density ${ }^{[55]}$. It is possible that in vivo, surveilling macrophages detect and phagocytize apoptotic bodies from osteoclasts before RANK on the surface can stimulate RANKL reverse 


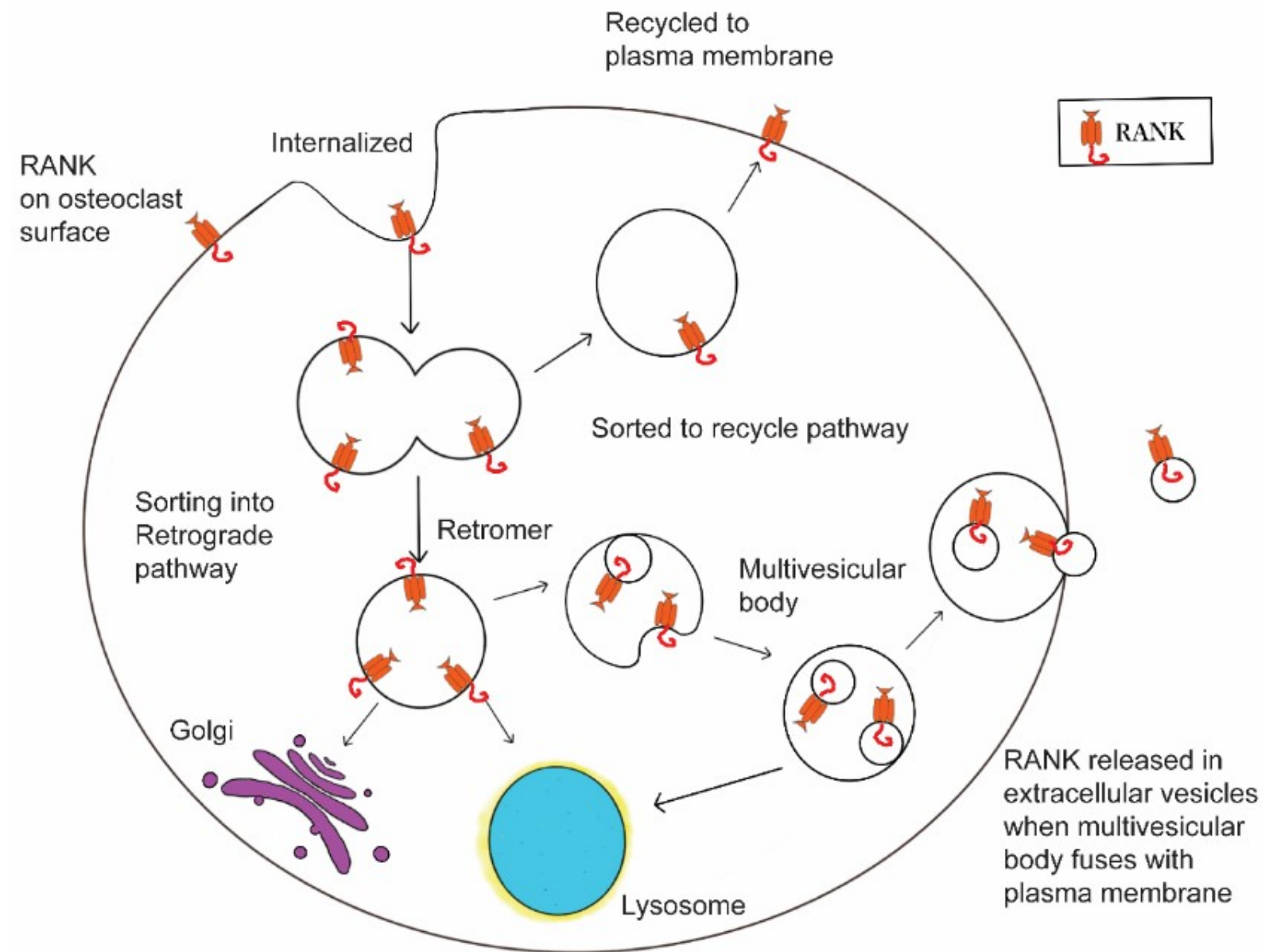

Figure 2. Regulation of RANK-containing EV production. We hypothesize that when RANK is internalized, an element of the normal receptor life cycle, it can either be recycled back to the plasma membrane, where it would be available for continued stimulation by RANKL. This would favor bone resorption. Alternatively, it could be sorted into a retrograde pathway that could lead to the Golgi for reuse of RANK, to the lysosome for degradation, or to multivesicular bodies, some of which would fuse with the plasma membrane and release RANK-EVs. All the retrograde pathways would remove RANK from the plasma membrane surface, and thus reduce stimulation by RANKL. In addition, shedding of RANK-EVs would also stimulate the RANKL reverse pathway and bone formation. If this idea is correct, shifts in the sorting of RANK could have profound effects on both bone resorption and bone formation, and understanding the underlying mechanisms may identify new strategies for treating bone disease.

signaling in cells of the osteoblast lineage ${ }^{[13]}$.

\section{RANKL-containing EVs and cancer invasion}

Numerous studies have suggested that cancer cells produce EVs that facilitate the growth and invasion of cancer cells to specific niches including bone ${ }^{[5,57]}$. Certain types of cancers, including breast, prostate, and multiple myeloma are particularly dangerous because of their ability to invade bone $e^{[57]}$. This is accomplished at least in part by sending signals that trigger inappropriate bone resorption by osteoclasts ${ }^{[57]}$. The "seed and soil" hypothesis proposes that that resorptive activity produces factors that trigger cancer growth and invasion and the stimulation of even more inappropriate bone resorption ${ }^{[57]}$. Might such cancer cells produce EVs containing RANKL to trigger bone resorption?

A recent study reports that EVs released by the breast cancer cell line MDA-MB-231 release EVs that contain the mRNA for RANKL ${ }^{[58]}$. However, RANKL was not detected by Western blot analysis, and cytofluorometric analysis also failed to convincingly demonstrate RANKL. These EVs were able to reach the 
bone microenvironment after intraperitoneal injection and be taken up by osteoclasts and osteoblasts. It was suggested that these EVs might be involved in deregulation of normal bone and endothelial physiology ${ }^{[5]}$.

\section{RANKL- and RANK- containing EVs outside of the skeletal system}

As mentioned above, RANKL and RANK are found in tissues other than the skeletal system. There is some evidence that these cells may release and be regulated by EVs containing these proteins. One study identified RANKL and RANK in circulating EVs in humans ${ }^{[43]}$. They found that the level of RANK in the circulating EVs served as a biomarker for psoriatic, but not rheumatoid, arthritis. They also found that isolated circulating EVs could regulate bone cells in vitro. Subsequently, it was shown that circulating RANK- and RANKL- containing EVs serve as biomarkers for bone loss linked to antiviral treatment for HIV-infections ${ }^{[44]}$.

Might the RANKL in circulating EVs prove to be a better biomarker than overall circulating RANKL? Numerous studies have examined RANKL in circulation and in other body fluids as a possible biomarker for disease, with limited success ${ }^{[59-65]}$. These were performed under the assumption that circulating RANKL would all be the external domain cleaved by sheddases. However, we now know that some portion of these molecules is present in EVs. The absolute amount of these molecules in EVs, or ratios, like the amount of circulating cleaved RANKL divided by RANKL in EVs, may prove useful as biomarkers.

As described above, the RANKL/RANK systems are regulators in the brain ${ }^{[23]}$. For example, injection of RANKL into the brain causes thermoregulatory disruption in female mice ${ }^{[21]}$. EVs have been shown to pass the blood brain barrier, particularly under inflammatory conditions. It is possible that EVs in circulation that are derived from the brain can be isolated based on specific neuronal, or microglial markers, and then assayed for RANKL and RANK. These may prove useful biomarkers for neuroinflammatory or neurodegenerative diseases.

Finally, circulating RANKL has been shown to be a biomarker for cardiovascular disease ${ }^{[63]}$. Detection of circulating RANKL in EVs and perhaps circulating RANK in EVs, may improve the diagnostic performance.

\section{CONCLUSIONS}

RANKL and RANK have been studied with great intensity since the discovery that RANKL was essential for stimulating osteoclast formation and bone resorption ${ }^{[6]}$. The therapeutic agent denosumab, a humanized monoclonal antibody that binds RANKL, has been successful for a decade for the treatment of osteoporosis and metastatic bone cancer ${ }^{[8,9]}$. RANKL and RANK have only recently been identified in EVs, and this area of study is still in its infancy. Already strong support for the hypothesis that RANKL and RANK-containing EVs have important roles in maintaining bone has emerged, as has evidence that these signaling molecules and their interactions can be targeted for therapeutics ${ }^{[24]}$ and used diagnostically ${ }^{[2,44]}$. The most exciting result so far has been the demonstration that certain crosslinked antibodies against RANKL can both inhibit bone resorption and stimulate bone formation ${ }^{[24]}$. Such bifunctional therapeutics might transform the treatment of bone diseases.

\section{DECLARATIONS}

\section{Authors' contributions}

Wrote first draft of manuscript, performed literature searches, and made initial interpretation: Holliday LS Constructed figures and aided in revision of manuscript: Patel SS 
Critically read, interpreted, and revised manuscript: Rody WJ Jr

\section{Availability of data and materials}

Not applicable.

\section{Financial support and sponsorship}

This work was supported by National Institutes of Health/National Institute of Dental and Craniofacial Research R21 DE019862 (Holliday LS), R03 DE027504 (Rody WJ Jr), a University of Florida College of Dentistry Seed Grant (Rody WJ Jr, Holliday LS), and a University Scholar Fellowship (Patel SS). The content is solely the responsibility of the authors and does not necessarily represent the official views of the National Institutes of Health.

\section{Conflicts of interest}

All authors declared that there are no conflicts of interest.

\section{Ethical approval and consent to participate}

Not applicable.

\section{Consent for publication}

Not applicable.

\section{Copyright}

(c) The Author(s) 2021.

\section{REFERENCES}

1. Boyce BF, Xing L. Functions of RANKL/RANK/OPG in bone modeling and remodeling. Arch Biochem Biophys 2008;473:139-46. DOI PubMed PMC

2. Lacey D, Timms E, Tan H, et al. Osteoprotegerin ligand is a cytokine that regulates osteoclast differentiation and activation. Cell 1998;93:165-76. DOI PubMed

3. Yasuda H, Shima N, Nakagawa N, et al. Osteoclast differentiation factor is a ligand for osteoprotegerin/osteoclastogenesis-inhibitory factor and is identical to TRANCE/RANKL. Proc Natl Acad Sci U S A 1998;95:3597-602. DOI PubMed PMC

4. Kong YY, Yoshida H, Sarosi I, et al. OPGL is a key regulator of osteoclastogenesis, lymphocyte development and lymph-node organogenesis. Nature 1999;397:315-23. DOI PubMed

5. Fata JE, Kong Y, Li J, et al. The Osteoclast differentiation factor osteoprotegerin-ligand is essential for mammary gland development. Cell 2000;103:41-50. DOI PubMed

6. Nagy V, Penninger JM. The RANKL-RANK story. Gerontology 2015;61:534-42. DOI PubMed

7. Compston JE, Mcclung MR, Leslie WD. Osteoporosis. Lancet 2019;393:364-76. DOI

8. Bone HG, Wagman RB, Brandi ML, et al. 10 years of denosumab treatment in postmenopausal women with osteoporosis: results from the phase 3 randomised FREEDOM trial and open-label extension. Lancet Diabetes Endocrinol 2017;5:513-23. DOI PubMed

9. Lipton A, Fizazi K, Stopeck AT, et al. Effect of denosumab versus zoledronic acid in preventing skeletal-related events in patients with bone metastases by baseline characteristics. Eur J Cancer 2016;53:75-83. DOI PubMed

10. Niel G, D’Angelo G, Raposo G. Shedding light on the cell biology of extracellular vesicles. Nat Rev Mol Cell Biol 2018;19:213-28. DOI PubMed

11. Mathieu M, Martin-Jaular L, Lavieu G, Théry C. Specificities of secretion and uptake of exosomes and other extracellular vesicles for cell-to-cell communication. Nat Cell Biol 2019;21:9-17. DOI PubMed

12. Théry C, Witwer KW, Aikawa E, et al. Minimal information for studies of extracellular vesicles 2018 (MISEV2018): a position statement of the international society for extracellular vesicles and update of the MISEV2014 guidelines. $J$ Extracell Vesicles 2018;7:1535750. DOI PubMed PMC

13. Caruso S, Poon IKH. Apoptotic cell-derived extracellular vesicles: more than just debris. Front Immunol 2018;9:1486. DOI PubMed PMC

14. Deng L, Wang Y, Peng Y, et al. Osteoblast-derived microvesicles: a novel mechanism for communication between osteoblasts and osteoclasts. Bone 2015;79:37-42. DOI PubMed

15. Huynh N, VonMoss L, Smith D, et al. Characterization of regulatory extracellular vesicles from osteoclasts. J Dent Res 2016;95:673-9. DOI PubMed PMC

16. Killock D. Bone: Osteocyte RANKL in bone homeostasis: a paradigm shift? Nat Rev Rheumatol 2011;7:619. DOI PubMed 
17. Nakashima T, Hayashi M, Fukunaga T, et al. Evidence for osteocyte regulation of bone homeostasis through RANKL expression. Nat Med 2011;17:1231-4. DOI PubMed

18. Xiong J, O'Brien CA. Osteocyte RANKL: new insights into the control of bone remodeling. J Bone Miner Res 2012;27:499-505. DOI PubMed PMC

19. Kong YY, Boyle WJ, Penninger JM. Osteoprotegerin ligand: a common link between osteoclastogenesis, lymph node formation and lymphocyte development. Immunol Cell Biol 1999;77:188-93. DOI PubMed

20. Kong Y, Boyle WJ, Penninger JM. Osteoprotegerin ligand: a regulator of immune responses and bone physiology. Immunology Today 2000;21:495-502. DOI PubMed

21. Hanada R, Leibbrandt A, Hanada T, et al. Central control of fever and female body temperature by RANKL/RANK. Nature 2009;462:505-9. DOI PubMed

22. Serrano EM, Ricofort RD, Zuo J, Ochotny N, Manolson MF, Holliday LS. Regulation of vacuolar H(+)-ATPase in microglia by RANKL. Biochem Biophys Res Commun 2009;389:193-7. DOI PubMed PMC

23. Hanada R. The role of the RANKL/RANK/OPG system in the central nervous systems (CNS). J Bone Miner Metab 2020. DOI PubMed

24. Ikebuchi Y, Aoki S, Honma M, et al. Coupling of bone resorption and formation by RANKL reverse signalling. Nature 2018;561:195200. DOI PubMed

25. Liu C, Walter TS, Huang P, et al. Structural and functional insights of RANKL-RANK interaction and signaling. J Immunol 2010;184:6910-9. DOI PubMed

26. Pan B, Farrugia AN, To LB, et al. The nitrogen-containing bisphosphonate, zoledronic acid, influences RANKL expression in human osteoblast-like cells by activating TNF-alpha converting enzyme (TACE). J Bone Miner Res 2004;19:147-54. DOI PubMed

27. Hikita A, Yana I, Wakeyama H, et al. Negative regulation of osteoclastogenesis by ectodomain shedding of receptor activator of NFkappaB ligand. J Biol Chem 2006;281:36846-55. DOI PubMed

28. Lynch CC, Hikosaka A, Acuff HB, et al. MMP-7 promotes prostate cancer-induced osteolysis via the solubilization of RANKL. Cancer Cell 2005;7:485-96. DOI PubMed

29. Asano T, Okamoto K, Nakai Y, et al. Soluble RANKL is physiologically dispensable but accelerates tumour metastasis to bone. Nat Metab 2019;1:868-75. DOI PubMed

30. Cappariello A, Loftus A, Muraca M, Maurizi A, Rucci N, Teti A. Osteoblast-derived extracellular vesicles are biological tools for the delivery of active molecules to bone. J Bone Miner Res 2018;33:517-33. DOI PubMed

31. Kobayashi-Sun J, Yamamori S, Kondo M, et al. Uptake of osteoblast-derived extracellular vesicles promotes the differentiation of osteoclasts in the zebrafish scale. Commun Biol 2020;3:190. DOI PubMed PMC

32. Huang X, Xiong X, Liu J, Zhao Z, Cen X. MicroRNAs-containing extracellular vesicles in bone remodeling: an emerging frontier. Life Sci 2020;254:117809. DOI PubMed

33. Eichholz KF, Woods I, Riffault M, et al. Human bone marrow stem/stromal cell osteogenesis is regulated via mechanically activated osteocyte-derived extracellular vesicles. Stem Cells Transl Med 2020;9:1431-47. DOI PubMed PMC

34. Morrell AE, Brown GN, Robinson ST, et al. Mechanically induced $\mathrm{Ca}^{2+}$ oscillations in osteocytes release extracellular vesicles and enhance bone formation. Bone Res 2018;6:6. DOI PubMed PMC

35. Qin Y, Peng Y, Zhao W, et al. Myostatin inhibits osteoblastic differentiation by suppressing osteocyte-derived exosomal microRNA218: A novel mechanism in muscle-bone communication. J Biol Chem 2017;292:11021-33. DOI PubMed PMC

36. Lv PY, Gao PF, Tian GJ, et al. Osteocyte-derived exosomes induced by mechanical strain promote human periodontal ligament stem cell proliferation and osteogenic differentiation via the miR-181b-5p/PTEN/AKT signaling pathway. Stem Cell Res Ther 2020;11:295. DOI PubMed PMC

37. Sato M, Suzuki T, Kawano M, Tamura M. Circulating osteocyte-derived exosomes contain miRNAs which are enriched in exosomes from MLO-Y4 cells. Biomed Rep 2017;6:223-31. DOI PubMed PMC

38. Yan Y, Wang L, Ge L, Pathak JL. Osteocyte-mediated translation of mechanical stimuli to cellular signaling and its role in bone and non-bone-related clinical complications. Curr Osteoporos Rep 2020;18:67-80. DOI PubMed

39. Holliday LS, McHugh KP, Zuo J, Aguirre JI, Neubert JK, Rody WJ Jr. Exosomes: novel regulators of bone remodelling and potential therapeutic agents for orthodontics. Orthod Craniofac Res 2017;20 Suppl 1:95-9. DOI PubMed PMC

40. Li D, Liu J, Guo B, et al. Osteoclast-derived exosomal miR-214-3p inhibits osteoblastic bone formation. Nat Commun 2016;7:10872. DOI PubMed PMC

41. Sun W, Zhao C, Li Y, et al. Osteoclast-derived microRNA-containing exosomes selectively inhibit osteoblast activity. Cell Discov 2016;2:16015. DOI PubMed PMC

42. Marton N, Kovács OT, Baricza E, et al. Extracellular vesicles regulate the human osteoclastogenesis: divergent roles in discrete inflammatory arthropathies. Cell Mol Life Sci 2017;74:3599-611. DOI PubMed

43. Okada E, Nakata H, Yamamoto M, Kasugai S, Kuroda S. Indirect osteoblast differentiation by liposomal clodronate. J Cell Mol Med 2018;22:1127-37. DOI PubMed PMC

44. de Menezes EG, Ramallho J, Bucovsky M, Shane E, Yin MT, Norris PJ. Serum extracellular vesicles expressing bone activity markers associate with bone loss after HIV antiretroviral therapy. AIDS 2020;34:351-61. DOI PubMed PMC

45. Sims NA, Martin TJ. Osteoclasts Provide Coupling signals to osteoblast lineage cells through multiple mechanisms. Annu Rev Physiol 2020;82:507-29. DOI PubMed

46. Rody WJ Jr, Chamberlain CA, Emory-Carter AK, et al. The proteome of extracellular vesicles released by clastic cells differs based on their substrate. PLoS One 2019;14:e0219602. DOI PubMed PMC 
47. Arana-Chavez VE, Bradaschia-Correa V. Clastic cells: mineralized tissue resorption in health and disease. Int J Biochem Cell Biol 2009;41:446-50. DOI PubMed

48. Wang Z, McCauley LK. Osteoclasts and odontoclasts: signaling pathways to development and disease. Oral Dis 2011;17:129-42. DOI PubMed

49. Linde A. Dentin matrix proteins: composition and possible functions in calcification. Anat Rec 1989;224:154-66. DOI PubMed

50. Bullock WA, Pavalko FM, Robling AG. Osteocytes and mechanical loading: the Wnt connection. Orthod Craniofac Res 2019;22 Suppl 1:175-9. DOI PubMed

51. Dodge T, Wanis M, Ayoub R, et al. Mechanical loading, damping, and load-driven bone formation in mouse tibiae. Bone 2012;51:8108. DOI PubMed PMC

52. Xia WF, Tang FL, Xiong L, et al. Vps35 loss promotes hyperresorptive osteoclastogenesis and osteoporosis via sustained RANKL signaling. J Cell Biol 2013;200:821-37. DOI PubMed PMC

53. Bourron O, Phan F, Diallo MH, et al. Circulating receptor activator of nuclear factor kB ligand and triglycerides are associated with progression of lower limb arterial calcification in type 2 diabetes: a prospective, observational cohort study. Cardiovasc Diabetol 2020;19:140. DOI PubMed PMC

54. Ma Q, Liang M, Wu Y, et al. Mature osteoclast-derived apoptotic bodies promote osteogenic differentiation via RANKL-mediated reverse signaling. J Biol Chem 2019;294:11240-7. DOI PubMed PMC

55. Stepan JJ, Alenfeld F, Boivin G, Feyen JH, Lakatos P. Mechanisms of action of antiresorptive therapies of postmenopausal osteoporosis. Endocr Regul 2003;37:225-38. PubMed

56. Pegtel DM, Gould SJ. Exosomes. Annu Rev Biochem 2019;88:487-514. DOI PubMed

57. Peinado H, Zhang H, Matei IR, et al. Pre-metastatic niches: organ-specific homes for metastases. Nat Rev Cancer 2017;17:302-17. DOI PubMed

58. Loftus A, Cappariello A, George C, et al. Extracellular vesicles from osteotropic breast cancer cells affect bone resident cells. J Bone Miner Res 2020;35:396-412. DOI PubMed

59. Dovio A, Data V, Angeli A. Circulating osteoprotegerin and soluble RANKL: do they have a future in clinical practice? J Endocrinol Invest 2005;28 Suppl 10:14-22. PubMed

60. Eleutherakis-Papaiakovou E, Kastritis E, Gavriatopoulou M, et al. Circulating soluble receptor activator of nuclear factor kappa B ligand and C-C motif ligand 3 correlate with survival in patients with waldenström macroglobulinemia. Clin Lymphoma Myeloma Leuk 2018;18:431-7. DOI PubMed

61. Granchi D, Garaventa A, Amato I, Paolucci P, Baldini N. Plasma levels of receptor activator of nuclear factor-kappaB ligand and osteoprotegerin in patients with neuroblastoma. Int J Cancer 2006;119:146-51. DOI PubMed

62. Oh KW, Rhee EJ, Lee WY, et al. Circulating osteoprotegerin and receptor activator of NF-kappaB ligand system are associated with bone metabolism in middle-aged males. Clin Endocrinol (Oxf) 2005;62:92-8. DOI PubMed

63. Raaz-Schrauder D, Schrauder MG, Stumpf C, et al. Plasma levels of sRANKL and OPG are associated with atherogenic cytokines in patients with intermediate cardiovascular risk. Heart Vessels 2017;32:1304-13. DOI PubMed

64. Sarink D, Schock H, Johnson T, et al. Receptor activator of nuclear factor kB ligand, osteoprotegerin, and risk of death following a breast cancer diagnosis: results from the EPIC cohort. BMC Cancer 2018;18:1010. DOI PubMed PMC

65. Zaman T, Sun P, Narod SA, Salmena L, Kotsopoulos J. Plasma RANKL levels are not associated with breast cancer risk in. BRCA1 2016;10:2475-83. DOI PubMed PMC 